\title{
Investigation of weak solar magnetic fields
}

\section{New observational results for the Srl $460.7 \mathrm{~nm}$ linear polarization and radiative transfer modeling}

\author{
M. Faurobert ${ }^{1}$, J. Arnaud ${ }^{2}$, J. Vigneau ${ }^{2}$, and H. Frisch ${ }^{1}$ \\ 1 Département Cassini, UMR 6529, Observatoire de la Côte d'Azur, BP 4229, 06304 Nice, France \\ 2 UMR 5572, Observatoire Midi-Pyrénées, 14 avenue Édouard Belin, 31400 Toulouse, France
}

Received 7 June 2001 / Accepted 3 August 2001

\begin{abstract}
Scattering polarization measurements were obtained with THEMIS in July 2000, close to the solar south Pole and to the east Equator and in a period of maximum solar activity. Using the THEMIS multi-lines spectro-polarimetric mode (MTR), we observed simultaneously four spectral domains containing the $460.7 \mathrm{~nm}$ Sr I line, several molecular lines around $515.9 \mathrm{~nm}$ and the $\mathrm{NaI} \mathrm{D}_{1}$ and $\mathrm{NaI} \mathrm{D}_{2}$ lines. This allows us to scan different altitudes in the solar atmosphere at the same time and provides us with a large set of constraints to study the behaviour of the magnetic field. This paper is devoted to the Sr I line which exhibits quite a strong linear polarization peak outside active regions. A detailed radiative transfer modeling is performed in order to interpret the observed center-to-limb variations of the line intensity and polarization. It was shown previously (Faurobert-Scholl 1993) that this line, which is sensitive to the Hanle effect, can be used as a diagnostic tool for the presence of weak turbulent magnetic fields in the solar photosphere outside active regions. The line polarization rates that we measured in July 2000 are 25\% lower than what has been reported previously, for observations near the minimum, or in the increasing phase, of the activity cycle (Stenflo et al. 1980). They are in agreement with other observations performed with a different observational set-up in August 2000 (Bommier \& Molodij 2001). We show that they are consistent with the presence of a weak turbulent magnetic field with an average strength between $20 \mathrm{G}$ and $30 \mathrm{G}$ in the upper solar photosphere. This is about twice the value which was derived from previous observations. This result raises the possiblity of a long-term variation of the turbulent photospheric magnetic field with the activity cycle.
\end{abstract}

Key words. techniques: polarimetric - techniques: spectroscopic - Sun: atmosphere - Sun: magnetic fields

\section{Introduction}

THEMIS has been especially designed for precise measurements of the vector magnetic field, simultaneously in several spectral bands chosen inside a large spectral domain. Its design minimizes instrumental polarization. See Arnaud et al. (1998) for a description of this telescope. It is thus particulary well suited for observations of linear scattering polarization outside active regions which forms the so-called second solar spectrum (Stenflo et al. 1997). There is no cross-talk from $I$ to the other Stokes parameters which is a condition for a precise determination of the polarization zero level. This is important as, near the limb, continuum polarization is no longer negligeable when compared to the polarization of most of the lines and it

Send offprint requests to: M. Faurobert,

e-mail: faurob@obs-nice.fr has to be taken into account to determine the polarization zero level. The absence of cross-talk makes also the polarization measurements insensitive to detector system non-linearities.

An atlas of the linear polarization of the solar spectrum observed close to the limb outside active regions has been published recently (Gandorfer 2000). It was obtained with the ZIMPOL II polarimeter at IRSOL in Locarno. It shows that a large number of spectral lines are linearly polarized with polarization features which are quite different to the intensity spectrum. A first presentation of the observations that we carried out at THEMIS in July 2000 for several spectral domains of the second solar spectrum is given in Arnaud et al. (2001). For most of the lines which are detected, the polarization mechanisms are still under debate. However, this is not the case for the now well understood polarization in the Sr I line, which 
exhibits one of the largest polarization of the second solar spectrum. This line is a normal triplet, and it is formed by multiple scattering in the solar photosphere. Its radiative transfer modeling has first been presented in detail in Faurobert-Scholl (1993) taking into account the depolarizing Hanle effect due to a weak turbulent magnetic field. This provides a diagnostic tool for turbulent magnetic fields which do not give rise to a measurable Zeeman effect because they have a mixed polarity at small scales. However the diagnostic relies on a precise calculation of the polarization rate expected in the absence of a magnetic field. This requires a good knowledge of the atmospheric model for quiet regions and precise values for the cross-section of depolarizing collisions. Atmospheric models are indeed reliable for the solar quiet photosphere and precise numerical calculations of collisional cross-sections have been performed for this line (see Faurobert-Scholl et al. 1995; Faurobert-Scholl 1996). The radiative transfer modeling has been controlled with the center-to-limb variations of the intensity profiles, which are not sensitive to weak magnetic fields.

In the following section we describe our observational set-up and we discuss some instrumental problems which limit the accuracy of the polarization measurements. We show that the main limitation is due to residual fringes which cannot be completely eliminated by the reduction procedure. Presently we estimate the accuracy on polarization rates to $0.5 \times 10^{-3}(0.05 \%)$, however the noise level is lower by a factor 10 . We give the center-to-limb variations of the polarization rate observed between one arcsec and 100 arcsec from the solar limb. The results are significantly smaller than those obtained at different periods of the activity cycle.

The third section is devoted to the radiative transfer modeling of the line intensity and linear polarization. The observed polarization rates are between $60 \%$ and $80 \%$ of the value which is expected in the absence of a magnetic field. We show that the center-to-limb variations of the depolarization reflect the depth-dependence of a "depolarization factor" which depends on the magnetic strength and on the depolarizing collision rate. The range of limb distances that were investigated gives access to the layer between $260 \mathrm{~km}$ and $380 \mathrm{~km}$ above the depth where $\tau_{5000}=1$. The depolarization is consistent with the presence of a weak turbulent magnetic field between $20 \mathrm{G}$ and $30 \mathrm{G}$ in this region.

\section{Observations}

\subsection{Observational procedure}

We report here on observations performed with THEMIS on July 2, 2000. The observations were made for slit positions very close to the solar south Pole for one part and to the east Equator for the other part, under excellent seeing conditions during the morning.

The polarization analysis package of THEMIS (cf. Paletou \& Molodij 2001) consists of two identical Fichou achromatic quarter-wave elements, made up of two plates in quartz and in $\mathrm{MgF}_{2}$, followed by a calcite beam splitter. Two beams of orthogonal polarization exit the polarimeter and go through the spectrometer for spectral analysis. They are recorded on one single CCD.

In principle the polarization signal is given by the difference between the two beams, but differences between the two optical paths and pixel to pixel variations of the sensitivity in the two parts of the detector will also contribute to the signal. However high precision spectropolarimetry can be achieved with such a system by applying the beam exchange technique (Semel 1994; Donati et al. 1990).

At THEMIS, up to summer 2000, the fast axis of the quater-wave plates could be oriented only at three different angular positions, namely at $0^{\circ}, 22.5^{\circ}$ and $45^{\circ}$. With this limitation the beam exchange technique was possible for one Stokes parameter only. We were interested in a precise determination of the center-to-limb variations of the Stokes parameter $Q$. This parameter is defined in a standard way, with respect to a system of axis parallel and perpendicular to the solar limb, so that positive $Q$ represents linear polarization parallel to the solar limb. In this reference system, for symmetry reasons, both $U$ and $V$ are zero in regions where there is no net magnetic flux. In order to perform beam exchange measurements for Stokes $Q$, with the available configuration of the quarter wave plates, we had to orientate the entrance slit in a direction making an angle of $45^{\circ}$ with the solar limb direction.

Four different polarization measurements were performed sequentially, $I \pm Q, I \pm U, I \pm V$ and $I \mp Q$ were recorded for the two beams. We used exposure times between 0.9 and $2 \mathrm{~s}$ and repeated the sequence at least 25 times to accumulate enough photons for the required sensitivity. The Stokes parameter $Q$ was obtained by combining the first and the fourth image of each beam. This avoids any spurious signal induced by differences between the two beams but, as the two images are taken with a time delay of a few seconds, we have to take into account seeing-induced cross talk from $I$ to $Q$.

Let us define the ratio of image 1 and image 4 , for each beam, denoted by $a$ and $b$ respectively,

$r_{a}=\frac{I+Q+\alpha_{1} I}{I-Q-\alpha_{2} I}, \quad r_{b}=\frac{I-Q-\alpha_{1} I}{I+Q+\alpha_{2} I}$.

The terms $\alpha_{1,2} I$ represent cross talk from $I$ to $Q$ at the two successive times where the images 1 and 4 were recorded. The linear polarization rate $p=Q / I$ is then obtained by

$p_{a, b}=\mp \frac{1-r_{a, b}}{1+r_{a, b}}$.

Notice that in the absence of cross talk these expressions give exactly the polarization rate $Q / I$, without any restriction to small polarization rates. Taking into account Eq. (1) and neglecting $\alpha_{1,2} I$ as compared to $I$ in the denominator, we obtain

$p_{a, b}=\frac{Q}{I} \pm \frac{\left(\alpha_{1}+\alpha_{2}\right)}{2}$. 
Then we averaged the results of the two beams to obtain the polarization rate as

$p=\frac{p_{a}+p_{b}}{2}$

This increases the signal-to-noise ratio and makes the signal free from the cross-talk terms which are eliminated.

The polarization rate $Q / I$ is determined from the ratio of images corresponding to the same beam but with two different configurations of the quarter wave plates. All the images were destretched so as to have the spatial resolution exactly along the detector columns and the spectral one along the rows. In principle with the method that we implemented it is unnecessary to correct the images for flat-field gain tables. However, this is not the case because the images are affected by the presence of interference fringes which are different for different positions of the quater wave plates and which slowly change with time. So we do have to correct each image by a flat-field table. This is described in the section devoted to data reduction.

We could not apply the beam exchange method to all the Stokes parameters because of the limitations of the first polarimeter on THEMIS. So more residual instrumental effects are present in the Stokes parameters, $U$ and $V$. In this paper, we present the results for $Q$.

As scattering polarization decreases very fast with limb distance it is important to precisely know the distance to the limb, as well as to take into account the image smearing. This is also required to be able to compare scattering polarization measurements obtained very close to the limb, at different times, in different observing conditions. When observing very near to it, we kept the solar limb inside the slit field of view. We define the limb position by the inflexion point in the continuum intensity profile at the limb. Its location in the image can be determined with an accuracy better than half a pixel. Taking into account the inclination of the entrance slit, one pixel represents 0.28 arcsec in the direction perpendicular to the limb. For limb distances larger than the slit length, where the limb is not on the image, we first take a short series of images at the limb and we rely on the polar coordinates given by the guiding system of the telescope to move the telescope radially to a given distance inside the disk. In order to increase the signal to noise ratio, we average over a small interval of 1 to 2 arcsec along the slit. This averaging has a similar smoothing effect as image motion and smearing: it reduces the spatial resolution in the direction perpendicular to the limb. The values of $\mu$ which are given in Table 1 are the mean values for each interval.

\subsection{Instrumental effects}

\subsubsection{Stray light}

To estimate the stray light we compared our observations to FTS observations published by Stenflo et al. (1983), FTS observations can be considered free from stray light. This comparison was done for the $460.7 \mathrm{~nm}$ Sr I line and for the $\mathrm{NaI} D_{2}$ line at $\mu=0.1$ which corresponds to a limb distance of 5 arcsec. A stray light upper limit of $1.5 \%$ of the continuum has been estimated for Sr I line and of less than $1 \%$ for the sodium line. On the other hand, the comparison of the observed intensity profiles with a radiative transfer modeling indicates that the stray ligth level slightly increases when $\mu$ decreases, reaching values of the order of $2 \%$ at $\mu=0.05$.

\subsubsection{Instrumental polarization}

No instrumental cross-talk among the Stokes parameters has been observed. We made some investigation of a possible depolarization due to birefringence occuring in the windows enclosing the helium filled tube of the telescope. In very clear sky conditions, frequent at Izania, sky brightness is mainly due to molecular Rayleigh scattering and is highly linearly polarized in the direction radial to the solar direction. In the red, the polarization rate can reach $70 \%$ to $80 \%$, at $90^{\circ}$ from the Sun (Coulson 1988). We measured this polarization, with a red filter with a $5 \mathrm{~nm}$ bandpass, simultaneously with THEMIS and with a small polarimeter fed with a $4 \mathrm{~cm}$ lens, pointed directly to the sky. The comparison of the results shows that, if any depolarization occurs, its level is below the accuracy of the measurements of our testing device (better than 5\%). Moreover, the comparison of our measurements with those performed at Locarno with Zimpol II (Gandorfer 2000) in the sodium lines (the strontium line was not in the observed domain with Zimpol) are in very good agreement so we are quite confident about the absence of any depolarization by the entrance and exit windows of THEMIS.

\subsubsection{Fringes}

With many plane parallel optical surfaces, fringes are unavoidable. They change when the retarders rotate so they do not cancel out while computing the Stokes parameters. Flat-fielding, in principle not necessary with the beam exchange technique, is here in fact very useful as it removes the fringes to a large extent, but not completly, as they are not perfectly stable.

The peak to peak amplitude of their residual after flatfielding is of the order of $10^{-2}$ of the continuum intensity $\left(I_{\mathrm{c}}\right)$ at $460 \mathrm{~nm}$. They can be afterwards reduced further, for instance by Fourier filtering. However, such data processing can also affect the signal. We shall describe in the following the procedure that we used to reduce the residual fringes at a level of $0.05 \%$. Fringes are presently an important limitation of the polarimetric precision.

\subsection{Data reduction procedure}

The classical photometric corrections, dark current subtraction and flat-field division, have been first applied to the data. The flat-field images have been built up from scans made while the telescope was moving in a way to 
have the solar image describing a pseudo-ellipse centered near the disc center. The telescope pointing direction and the angular size of the pseudo-ellipse were chosen to avoid active regions across the slit field of view. This was not an easy task, the Sun being very active at this time. The images of the flat-field scans were stacked for each of the four retarder positions used for the observations.

The four resulting flat-field images, corresponding to the four positions of the retarder, contain spectral features along the row axis, that have to be removed in order to get flat-field gain tables. This is achieved in a standard way. Each image is divided by the mean spectrum obtained by averaging over the rows. This provides four flat-field gain tables where one can see several fringes systems, with various orientations and interfringe spacing. However, we do not get in these tables the components of the fringes which lie along the row axis because they are removed when we divide by the average spectrum. When dividing the near limb images by the flat-field gain tables, fringes are reduced by about one order of magnitude (they do not completly vanish because they are not perfectly stable). The flat-fielded images are then recentered in the spectral direction (along the rows) and when it was possible to use the limb for this purpose, also in the spatial one, in a way to limit image smearing due to image motions and pointing drifts. Then the polarization is extracted as explained above. As flat-fielding does not correct the fringes along the row axis, we get a modulation, of the order of $0.6 \%$ peak to peak, added to the $Q / I$ profiles (see Fig. 1).

We used the following method to reduce it to an acceptable level. Let us consider the images that are used to obtain the flat-field gain tables; we can compute pseudo $Q / I$ profiles by using Eqs. (1) to (4). They should be zero because the flat-field images are obtained in such a way that they should not be polarized. The pseudo $Q / I$ signal that we obtain is due to the presence of fringes and possibly other instrumental effects. So the average spectra obtained by averaging over the rows contain the components of the fringes along the spectral axis. These pseudo mean $Q / I$ profiles are subtracted from the $Q / I$ profiles obtained for the data. The result of this correction procedure is shown in Fig. 1 for the SrI line. The modulation of the polarization profile appears clearly on the dot-dashed curve. Its is quite well removed when one subtracts the fringe present in the flat-field average row, as explained previously. The residual fringes that we can see in the continuum have a peak to peak amplitude of about $0.05 \%$.

\subsection{Results}

The Strontium I $460.7 \mathrm{~nm}$ line has one of the largest polarization rates of the second solar spectrum. Its linear polarization has been measured first by Wiehr (1978). It has been extensively observed by Stenflo and co-workers who reported on its center to limb variations (Stenflo et al. 1980) as well as on a variability of the polarization

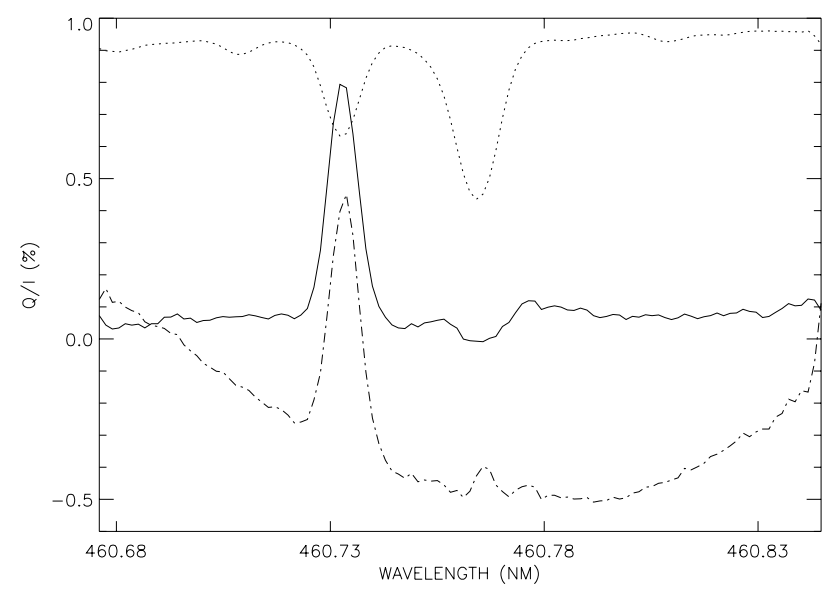

Fig. 1. $I$ (dotted line) and $Q / I$ profiles at $\mu=0.1$. The dasheddotted line represents the $Q / I$ profile before correction of the fringe present in the flat-field average row. About one half period of this fringe is visible in the wavelength range of the figure. Its peak to peak amplitude is about $1 \%$. The flat-field correction allows to reduce by one order of magnitude the amplitude of all the fringes, except for this one. The full line displays the $Q / I$ profile after subtraction of the flat field "pseudo $Q / I$ ", as explained in the text. It can be noticed that one effect of this fringe, which is also corrected, is a weak spurious positive $Q$ signal in the $460.76 \mathrm{~nm}$ FeI line in place of the actual continuum depolarization.

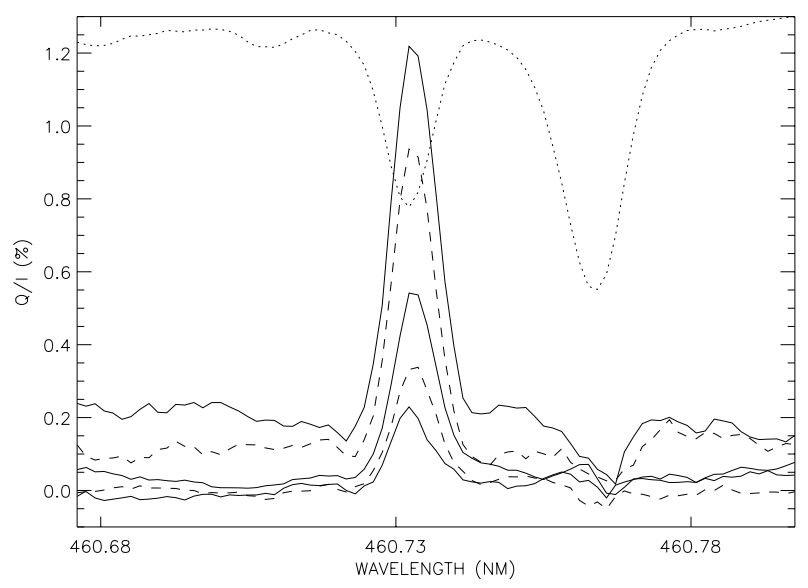

Fig. 2. Polarization profiles of the SrI $460.73 \mathrm{~nm}$ at 5 distances from the solar limb: $1.3 \operatorname{arcsec}(\mu=0.05)$, full line; $5 \operatorname{arcsec}(\mu=0.10)$, dashed line; $19.5 \operatorname{arcsec}(\mu=0.20)$, full line; $50 \operatorname{arcsec}(\mu=0.32)$, dashed line; $96 \operatorname{arcsec}(\mu=0.44)$, full line and the intensity at $\mu=0.20$ in arbitrary units (dotted line). The continuum depolarization in the FeI $460.76 \mathrm{~nm}$ line is visible near the solar limb. Fringe residuals make the continuum polarization difficult to measure. The $Q / I$ continuum level is determined as explained in the text.

amplitude (Stenflo et al. 1997), which is likely due to spatially changing Hanle depolarization.

The ZIMPOL 1994 and the IRSOL 1995 observations reported by Stenflo et al. (1997) display polarization values between $1.55 \%$ and $1.95 \%$ very near to the limb and between $0.7 \%$ and $1.05 \%$ at $\mu=0.20(\mu=\cos \theta)$. 
Table 1. Variations with $\mu$ of $Q / I$, in units of $10^{-4}$ for the $406.7 \mathrm{~nm}$ Sr I line center and continuum.

\begin{tabular}{ccc}
\hline$\mu$ & $\begin{array}{c}\text { Sr I } \\
460.73 \mathrm{~nm}\end{array}$ & $\begin{array}{c}\text { Cont. } \\
460 \mathrm{~nm}\end{array}$ \\
\hline 0.05 & 123 & 19 \\
0.08 & 107 & 14 \\
0.10 & 95 & 11 \\
0.13 & 81 & 9 \\
0.15 & 69 & 7.4 \\
0.18 & 61 & 6.2 \\
0.20 & 53 & 5.4 \\
0.23 & 46 & 4.6 \\
0.29 & 39 & 3.4 \\
0.32 & 37 & 2.9 \\
0.35 & 33 & 2.6 \\
0.44 & 23 & 1.8 \\
\hline \hline
\end{tabular}

We measured polarization values of $1.23 \%$ at $\mu=0.05$ and of $0.53 \%$ at $\mu=0.2$. They are in good agreement with other recent THEMIS observations (Bommier \& Molodij 2001).

Those differences may come from magnetic field variations inducing different Hanle depolarizations or from other physical changes in the solar atmosphere.

Figure 2 shows the $Q / I$ polarization profiles that we derived for 5 limb distances, and Table1 gives the value of the line core polarization that we obtain for all the observed limb distances.

The $Q / I$ continuum level is, as it is visible in Fig. 2, too much affected by fringe residuals to be accurately measured. So we determined an average continuum polarization level (by a simple averaging over the fringe residuals) and we adjusted its value to the one which is calculated with the radiative transfer modeling described in the following section. The calculated continuum polarization is given in Table 1. To obtain the value of the polarization peak we first measure the amplitude of the peak above the average continuum level and we add it to the continuum polarization derived from the model. The inaccuracy in the determination of the average continuum level, due to fringe residuals, is thus the main source of inaccuracy in the value of the polarization peak.

\section{Radiative transfer modeling}

The SrI line at $460.7 \mathrm{~nm}$ is a resonance line and a normal triplet. Its radiative de-excitation rate is quite large $\Gamma_{\mathrm{R}}=2.01 \times 10^{8} \mathrm{~s}^{-1}$. Therefore, although it is formed in the photosphere, radiative transitions dominate over collisional transitions. Photon scattering is thus the dominant mechanism for the line formation. The intensity and polarization profiles, together with continuum intensity and polarization, are calculated as described in FaurobertScholl (1993) and Faurobert-Scholl et al. (1995). Let us first briefly recall the main steps of these calculations.

\subsection{Method of modeling}

We proceed in two steps. First, the line and continuum intensities are obtained by a non-LTE caculation where the polarization is neglected. For that we use the MULTI code of Carlson with a quite simple atomic model for the Strontium atom (see Faurobert-Scholl 1993). This provides us with the line optical depth scale as a function of the altitude in the photosphere and allows us to calculate creation and destruction coefficients for the equivalent two-level atom form of the line source function. This twolevel atom formalism is then used to calculate the scattering polarization. This is justified because the line is mainly formed by absorptions and emissions between the upper and lower atomic levels of the transition. The polarized line source function is a two-component vector for the two Stokes parameter $I$ and $Q$ ( $U$ and $V$ are vanishing in the absence of a net magnetic vector), namely

$\boldsymbol{S}_{\mathrm{L}}(\tau, x, \Omega)=\left[\boldsymbol{J}_{\mathrm{sc}}+\left(\varepsilon^{\prime} \mathcal{B}+\eta B^{*}\right) \boldsymbol{U}\right] /\left(1+\varepsilon^{\prime}+\eta\right)$,

where $\boldsymbol{J}_{\mathrm{sc}}$ denotes the scattering term, $\mathcal{B}$ is the Planck function and $\boldsymbol{U}$ is the vector $(1,0)^{\dagger}$. As usual $\tau$ is the line integrated optical depth, $x$ is the frequency measured from line center in Doppler width units and $\Omega$ is the propagation direction of photons. The expression (5) has the same form as for a two-level atom with an additional creation term $\eta B^{*}$ and a sink term $\eta$ which are due to the coupling with the continuum.

Scattering of photons leads to both partial frequency redistribution and linear polarization. The scattering term is given by

$$
\begin{aligned}
\boldsymbol{J}_{\mathrm{sc}}(\tau, x, \Omega)=\frac{1}{\phi(x)} \int_{-\infty}^{+\infty} \mathrm{d} x^{\prime} \int \frac{\mathrm{d} \Omega^{\prime}}{4 \pi} \\
\hat{R}\left(x, x^{\prime}, \Omega, \Omega^{\prime}, \boldsymbol{B}\right) \boldsymbol{I}\left(\tau, x^{\prime}, \Omega^{\prime}\right),
\end{aligned}
$$

where $\hat{R}$ is the redistribution matrix, $\boldsymbol{I}$ is the Stokes vector of the radiation field, $\boldsymbol{I}=(I, Q)^{\dagger}$ and $\phi(x)$ is the absorption profile. The exact derivation of the redistribution matrix in the presence of magnetic fields and collisions was achieved recently by Bommier (1997a,b) (see also Bommier \& Stenflo 1999 for an illustrative classical approach). It leads to complex analytical expressions for the redistribution matrix, because frequency redistribution is coupled to angular and polarization redistribution.

The behaviour of the redistribution matrix depends on 2D frequency domains referred to as the "core" and the "wing" domains whose boundaries depend both on $x$ and $x^{\prime}$. The definition of these domains is given in Bommier (1997b) (see also Faurobert et al. 2001). However it was shown numerically that in non-magnetic media or in the presence of a turbulent magnetic field angle-average approximations may be safely used (see Faurobert et al. 2001). Moreover, in these cases one can also use a simple definition of the core domain as $x \leq x_{\mathrm{c}}$, where $x_{\mathrm{c}}$ is a cut off frequency of the order of a few Doppler widths and $x$ is the emergent frequency (see Faurobert et al. 1999). In each domain, angular and polarization redistribution are decoupled from frequency redistribution. 
For a normal triplet, in the line core, we have

$$
\begin{aligned}
\hat{R}\left(x, x^{\prime}, \Omega, \Omega^{\prime}\right)= & {\left[\gamma R_{\mathrm{II}}\left(x, x^{\prime}\right)+b R_{\mathrm{III}}\left(x, x^{\prime}\right)\right] \hat{P}_{\mathrm{H}}\left(\Omega, \Omega^{\prime}, \boldsymbol{B}\right) } \\
& +c R_{\mathrm{III}}\left(x, x^{\prime}\right) \hat{P}_{\mathrm{is}},
\end{aligned}
$$

where $\hat{P}_{\mathrm{H}}$ denotes the Hanle phase matrix and $\hat{P}_{\text {is }}$ the isotropic phase matrix; $\gamma, b$ and $c$ are branching ratio coefficients which depend on the radiative and collisional transition rates. Their expressions are given in FaurobertScholl (1993). $R_{\mathrm{II}}$ and $R_{\mathrm{III}}$ are angle-averaged frequency redistribution functions referring respectively to coherent and incoherent scattering. In the wing domain we have a similar expression but the Hanle phase matrix is replaced by the Rayleigh phase matrix.

In the presence of a weak turbulent magnetic field, the Hanle phase matrix reduces to

$\hat{P}_{\mathrm{H}}\left(\Omega, \Omega^{\prime}, \boldsymbol{B}\right)=\hat{P}_{\mathrm{is}}+\frac{3}{8} W_{2} W_{B} \hat{P}^{(2)}\left(\Omega, \Omega^{\prime}\right)$.

In this equation $W_{2}$ is a numerical constant which depends on the quantum numbers $J$ and $J^{\prime}$ of the upper and lower levels of the line transition. It is equal to unity for a normal triplet. $W_{B}$ is a depolarization factor which depends on the average strength of the turbulent magnetic field and on the depolarizing collision rate. It is given by

$W_{B}=1-0.4\left(\frac{\gamma_{\mathrm{H}}^{2}}{1+\gamma_{\mathrm{H}}^{2}}+\frac{4 \gamma_{\mathrm{H}}^{2}}{1+4 \gamma_{\mathrm{H}}^{2}}\right)$,

where $\gamma_{\mathrm{H}}$ is the so-called Hanle parameter,

$\gamma_{\mathrm{H}}=0.88 g_{J} \frac{B}{\Gamma_{\mathrm{R}}+D^{(2)}}$,

where $g_{J}$ is the Landé factor of the upper level, $\Gamma_{\mathrm{R}}$ is the radiative transition rate and $D^{(2)}$ is the depolarizing collision rate, both given in units of $10^{7} \mathrm{~s}^{-1}$; for the SrI $460.7 \mathrm{~nm}$ line $\Gamma_{\mathrm{R}}=20.1$ in this unit. The sensitivity domain of the line to the Hanle effect corresponds to $\gamma_{H}=1$, i.e. to magnetic fields of about 20 G. Depolarizing collisions are mainly due to neutral hydrogen. We recall that the collision rate may be written as (see Faurobert-Scholl et al. 1995)

$D^{(2)}=1.37 N_{\mathrm{H}}(T / 5000)^{0.16}$,

where $N_{\mathrm{H}}$ is the neutral hydrogen density and $T$ the temperature.

In a plane parallel atmosphere with a turbulent isotropic magnetic field, the radiation field is symmetrical with respect to the vertical direction. The matrix $\hat{P}^{(2)}\left(\Omega, \Omega^{\prime}\right)$ reduces to a $2 \times 2$ matrix denoted by $\hat{P}_{0}^{(2)}\left(\mu, \mu^{\prime}\right)$ which depends only on the cosines $\mu$ and $\mu^{\prime}$ of colatitudes for the incoming and outgoing directions

$$
\begin{array}{lll}
\hat{P}_{0}^{(2)} & \left(\mu, \mu^{\prime}\right)= \\
& \frac{1}{2}\left(\begin{array}{cc}
\left(\frac{1}{3}-\mu^{2}\right)\left(1-3 \mu^{\prime 2}\right) & \left(1-3 \mu^{2}\right)\left(1-\mu^{\prime 2}\right) \\
\left(1-\mu^{2}\right)\left(1-3 \mu^{\prime 2}\right) & 3\left(1-\mu^{2}\right)\left(1-\mu^{\prime 2}\right)
\end{array}\right)
\end{array}
$$

The isotropic phase matrix has only one non-zero element, $\hat{P}_{\text {is }}(1,1)=1$. This matrix does not give rise to polarization.
We see from Eqs. (8) to (10) that the phase matrix is very similar to the Rayleigh phase matrix except for the additional depolarization factor $W_{B}$ which is equal to unity when $B=0$ and smaller that one in the presence of a turbulent magnetic field.

We also take into account the polarization in the continuum spectrum, which is due to Thomson scattering by free electrons and to Rayleigh scattering by hydrogen molecules. For both processes the scattering phase matrix is of the Rayleigh type. The continuum source function is written

$$
\begin{aligned}
\boldsymbol{S}_{\mathrm{c}}=\left[k_{\mathrm{a}} \mathcal{B} \boldsymbol{U}+k_{\mathrm{sc}}\right. & \int_{-\infty}^{+\infty} \mathrm{d} x^{\prime} \int \frac{\mathrm{d} \Omega^{\prime}}{4 \pi} \\
& \left.\hat{P}_{\mathrm{R}}\left(\Omega^{\prime}, \Omega\right) \boldsymbol{I}\left(\tau, x^{\prime}, \Omega^{\prime}\right)\right] /\left(k_{\mathrm{a}}+k_{\mathrm{sc}}\right),
\end{aligned}
$$

where $k_{\mathrm{a}}$ is the continuum absorption coefficient and $k_{\mathrm{sc}}$ is the scattering coefficient.

The scattering and absorption coefficients of the quiet solar atmosphere in the line frequency range are calculated as in MULTI with the Uppsala opacity package.

\subsection{Intensity and polarization profiles}

We use the atmospheric model of Fontenla et al. (1993, Model C) with a microturbulent velocity of $1 \mathrm{~km}^{-1}$ in the upper photosphere. The polarized radiative transfer equation with partial frequency redistribution is solved as described in Faurobert-Scholl (1993). The intensity and polarization profiles are convolved with a Gaussian profile in order to simulate the smearing effect of macroturbulent velocities in the photosphere and finite spectral resolution. A good fitting of the intensity profiles observed close to the solar limb is achieved with macroturbulent velocities of $2.5 \mathrm{~km}^{-1}$.

Figure 3 shows the comparison between the calculation and the intensity profiles observed at $\mu=0.05,0.10,0.18$ and 0.44 . For $\mu=0.05$ we had to take into account a fraction of stray light of $2 \%$ of the continuum intensity in order to get a good fit of the intensity in the line core.

Figure 4 is devoted to the linear polarization profiles. We compare the observed profiles with those which are derived from the model without any magnetic field. This shows that the observed polarization rates are smaller than the polarization calculated with a non-magnetic model.

In the following we shall assume that the depolarization is due to the Hanle effect of a turbulent weak magnetic field. Figure 5 shows the same quantities as in Fig. 4, but for a model that takes into account the depolarizing Hanle effect due to a depth-independent turbulent magnetic field of $25 \mathrm{G}$. Quite a good agreement with the observed profiles is now obtained.

\subsection{Sensitivity of the diagnostics to the magnetic strength}

In this section we address the question of the sensitivity of the Hanle effect diagnostics. First, we define the 


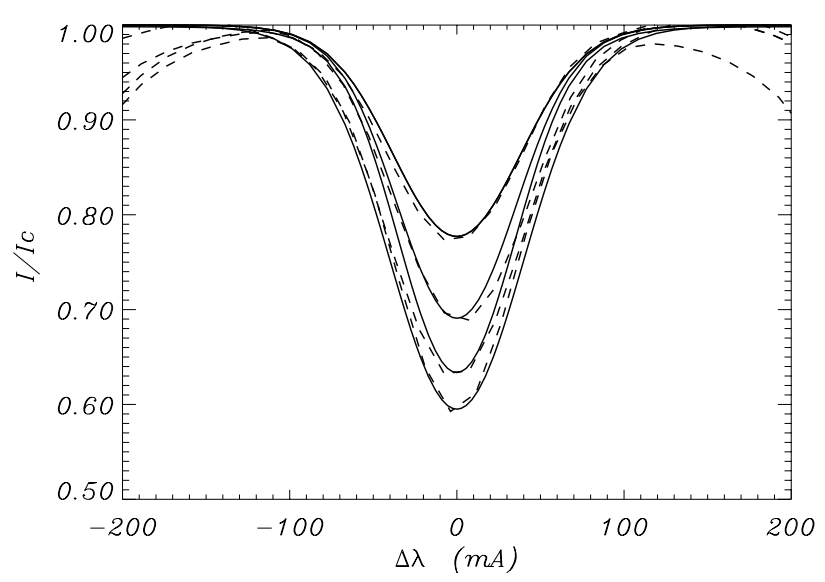

Fig. 3. Intensity profiles of the $\mathrm{Sr}$ I $460.73 \mathrm{~nm}$ line at 4 distances from the solar limb: $\mu=0.05,0.1,0.18$ and 0.44 . The dashed lines refer to the observed profiles, full lines show the results of the radiative transfer modeling.

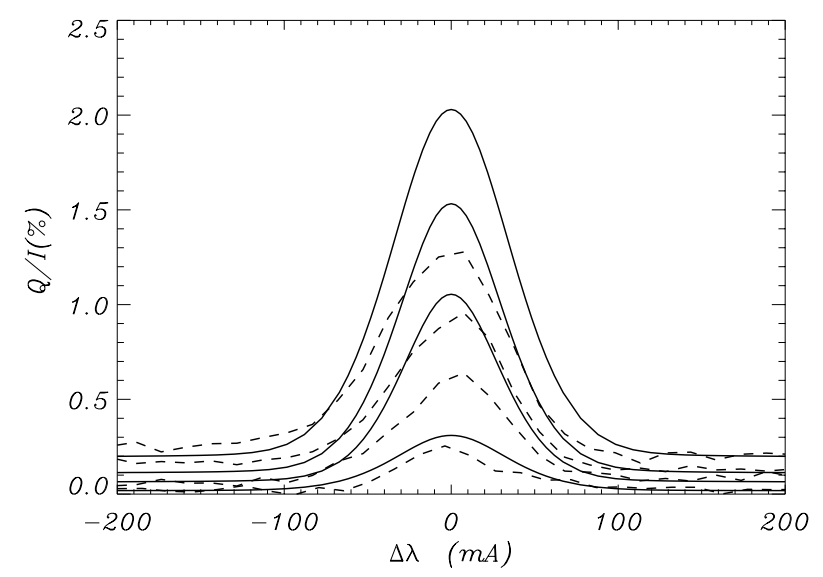

Fig. 4. Polarization profiles of the Sr I $460.73 \mathrm{~nm}$ line at 4 distances from the solar limb: $\mu=0.05,0.1,0.18$ and 0.44 . The dashed lines refer to the observed profiles, full lines show the results of the radiative transfer modeling with $B=0$.

depolarization as the ratio of the line core polarization in the presence of a magnetic field and of the non-magnetic polarization, $\left\langle Q_{B}>/<Q_{0}>\right.$. The brackets mean that we consider an average value of the polarization over a frequency band in the line core. We checked that the depolarization is not very sensitive to the width of the frequency band if we keep it smaller or equal to the width of the line core. The results which are shown in the following are obtained for a band-width of $40 \mathrm{~m} \AA$. This averaging is aimed at smoothing the observed profiles over several pixels of the frequency grid (the pixel size is $15 \mathrm{~m} \AA$ ).

The Hanle effect does not depend on frequency, as shown by Eq. (8). The effect of a turbulent magnetic field on the phase matrix is contained in the coefficient $W_{B}$ which multiplies with the matrix $\hat{P}_{0}^{(2)}$. Thus, in the case where the emergent polarization is created by one single scattering in the atmosphere the depolarization is equal to the value of $W_{B}$ at the depth point where the scattering occurs. The depolarization is then sensitive to the

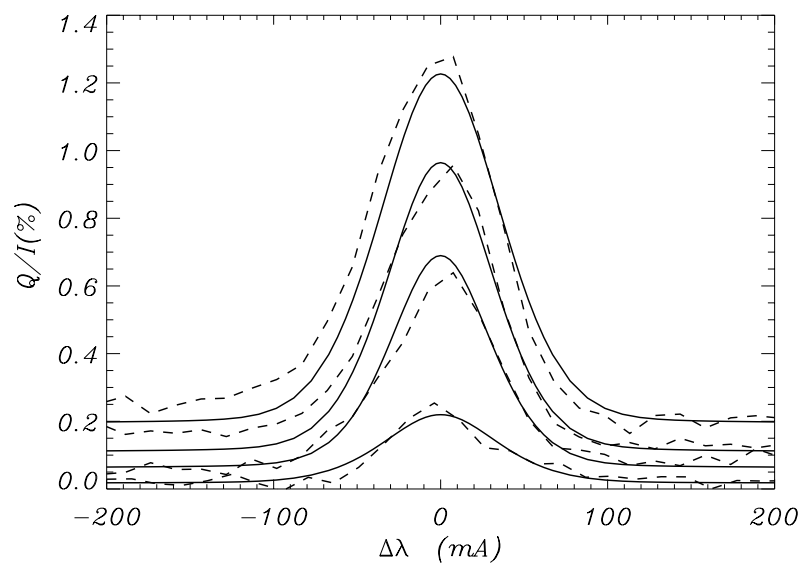

Fig. 5. Polarization profiles of the Sr I line. Same as in Fig. 4 but for $B=25 \mathrm{G}$.

value of the magnetic field at the corresponding depth. The Strontium line is formed by multiple scattering, but this is not a very thick line so the number of scatterings by line photons in the photosphere is small. Figure 6 shows that $\left.\left\langle Q_{B}\right\rangle /<Q_{0}\right\rangle \simeq W_{B}(\tau=\mu)$, i.e. that the depolarization as a function of $\mu$ is very close to the coefficient $W_{B}$ as a function of the line optical depth $\tau$. The reason is that $\left\langle Q_{B}\right\rangle$ and $\left\langle Q_{0}\right\rangle$ scale as the second component of the emissivity vector defined in Eq. (6), which is proportional to $W_{B}$. As we already noticed, in the presence of a turbulent magnetic field, the Hanle phase matrix is identical to the Rayleigh phase matrix, except for the depolarizing factor $W_{B}$, so the ratio $\left\langle Q_{B}>/<Q_{0}>\right.$ takes the value of $W_{B}$ at the optical depths where the emergent polarization is formed. For values of $\mu$ between 0.05 and 0.44 the corresponding depths are between $380 \mathrm{~km}$ and $260 \mathrm{~km}$ above the depth where $\tau_{5000}=1$.

The depolarizing coefficient $W_{B}$ is related to the Hanle parameter $\gamma_{\mathrm{H}}$ which depends on the square of the magnetic strength. We checked that the response function of the depolarization to a small variation of $B^{2}$ at one point in the atmosphere is a very narrow peak localized at the perturbation depth. This is not a surprising result because it is well known that the Hanle effect is a local effect.

The observed points are shown on the lower panel of Fig. 6. Their center-to-limb variations are well represented by the Hanle depolarization due to turbulent magnetic fields between $20 \mathrm{G}$ and $30 \mathrm{G}$ located in the upper photosphere, between $260 \mathrm{~km}$ and $380 \mathrm{~km}$. The scatter of the observed points does not allow us to measure any gradient of the magnetic strength in this layer.

\section{Conclusions}

The results which are presented here lead to the question of a possible variation of the turbulent photospheric magnetic field with the solar activity cycle. The origin of the turbulent field is still under debate (Cattaneo 1999). It could be due either to a local small scale dynamo in the photosphere, or to an alpha-omega dynamo acting just 

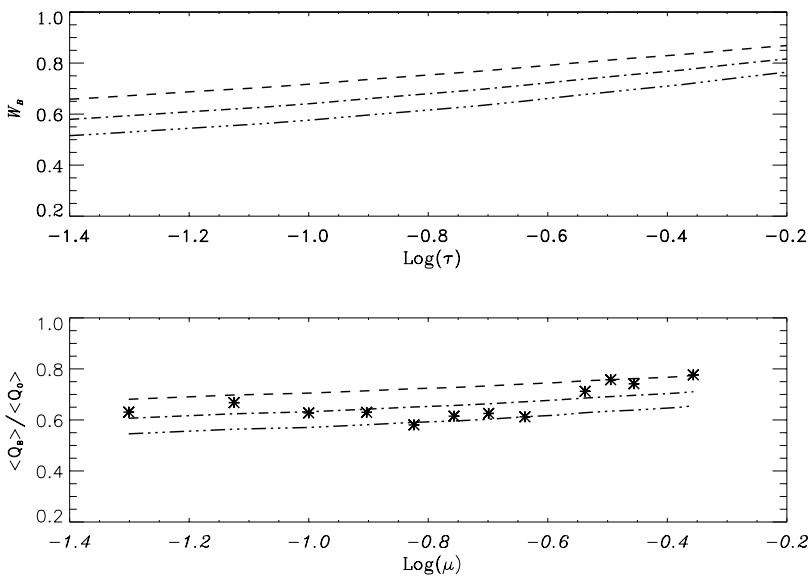

Fig. 6. Upper panel: variations of the depolarization coefficient $W_{B}$ defined in Eq. (9) as function of the line optical thickness $\tau$. The selected range for $\tau$ corresponds to the values of $\mu$ where the line depolarization has been measured. The three curves correspond to three values of the magnetic strength $B$, dashed line: $B=20 \mathrm{G}$, dot-dashed line: $B=25 \mathrm{G}$, doubledot-dashed line: $B=30 \mathrm{G}$. Lower panel: variation of the line depolarization as a function of $\mu$ for the same three values of $B$. The asterisks show the observed points.

below the photosphere. It could also be the result of the dissipation of the magnetic fields of sunspots by an effective magnetic diffusivity. This third mechanism would certainly contribute to an increase of the turbulent field during the maxima of solar activity. But the other two mechanisms could also be enhanced during activity maxima. Global changes in the dynamics and thermodynamics of the Sun are observed in correlation with the activity cycle, affecting for example the solar radius and its luminosity. The so-called "quiet regions" are likely to be sensitive to such changes as well.

Observational studies of the long term variations of the photospheric turbulent magnetic field should be continued with a single well calibrated instrument in order to confirm this variability.

Acknowledgements. We thank the THEMIS technical staff for its help, particularly the telescope operators and, among them, O. Grassin for his dedication to the development of a user's interface which made observations a lot easier.
THEMIS is operated on the Island of Tenerife by CNRSCNR in the Spanish Observatorio del Teide of the Instituto de Astrofísica de Canarias.

\section{References}

Arnaud, J., Mein, P., \& Rayrole, J. 1998, in a Crossroads For European Solar and Heliographic Physics, Tenerife, March 1998, ed. ESA SSPG and IAC, ESA SP-417, 213

Arnaud, J., Faurobert, M., Vigneau, J., \& Paletou, F. 2001 in Advanced Solar Polarimetry - Theory, Observation and Instrumentation, ed. M. Sigwarth, ASP Conf. Ser., 236, 151

Bommier, V. 1997a, A\&A, 328, 706

Bommier, V. 1997b, A\&A, 328, 726

Bommier, V., \& Stenflo, J. O. 1999, A\&A, 350, 327

Bommier, V., \& Molodij, G. 2001, A\&A, submitted

Cattaneo, F. 1999, ApJ, 515, L39

Coulson, K.L. 1998, Polarization and intensity of light in the atmosphere (A. Deepak Publishing, Hampton, Virginia, USA)

Donati, J.-F., Semel, M., Rees, D. E, Taylor, K., \& Robinson, R. D. 1990, A\&A, 232, L1

Faurobert-Scholl, M. 1993, A\&A, 268, 765

Faurobert-Scholl, M. 1996, Solar Phys., 164, 79

Faurobert-Scholl, M., Feautrier, N., Machefert, F., Petrovay, K., \& Spielfiedel, A. 1995, A\&A, 298, 289

Faurobert-Scholl, M., Paletou, F., \& Bommier, V. 1999, in Solar Polarization, ed. K. N. Nagendra, \& J. O. Stenflo (Kluwer), 115

Faurobert, M., Frisch, H., \& Nagendra, K. 2001, in Magnetic Fields across the HR Diagram, ed. G. Mathys, S. Solanki, \& D. T. Wickramasinghe, ASP Conf. Ser., 248

Fontenla, J. M., Avrett, E. H., \& Loeser, R. 1993, ApJ, 406, 319

Gandorfer, A. 2000, The Second Solar Spectrum, a high spectral resolution polarimetric survey of scattering polarization at the solar limb in graphical representation (Hochschulverlag AG and der ETH, Zürich)

Paletou, F., \& Molodij, G. 2001, in Advanced Solar Polarimetry - Theory, Observation and Instrumentation, ed. M. Sigwarth, ASP Conf. Ser., 236, 9

Semel, M. 1994, in Solar Surface Magnetism, ed. R. J. Rutten, \& C. J. Schrijver (Kluwer)

Stenflo, J. O., Baur, T. G., \& Elmore, D. F. 1980, A\&A, 84, 60

Stenflo, J. O., Bianda, M., Keller, C. U., \& Solanki, S. K. 1997, A\&A, 322, 994

Stenflo, J. O., Twerenbold, D., Harvey, J. W., \& Brault, J. W. 1983, A\&AS, 54, 505

Wiehr, E. 1978, A\&A, 67, 257 